\title{
Detection of crystals in synovial fluids by light microscopy: sensitivity and reliability
}

\author{
CAROLINE GORDON, ANGELA SWAN, AND PAUL DIEPPE \\ From the Rheumatology Unit, University Department of Medicine, Bristol Royal Infirmary, Bristol
}

SUMmary Polarised light microscopy of synovial fluid is an established diagnostic technique widely regarded as reliable for the detection of crystals. The threshold concentration of crystals which can be detected has been investigated and the sensitivity and specificity of six observers compared. Various concentrations of laboratory manufactured crystals of monosodium urate monohydrate (MSUM), calcium pyrophosphate dihydrate (CPPD), and basic calcium phosphates (BCP) were added to the synovial fluid. The threshold for reliable identification of MSUM and CPPD was in the range of $10-100 \mu \mathrm{g} / \mathrm{ml}$. False positives were frequent. The mean sensitivity of the six observers for MSUM was $69 \%$ and for CPPD was $82 \%$. The mean specificity for MSUM was $97 \%$ and for CPPD was $78 \%$. There was much discrepancy in the results of the slides stained with alizarin red S. Thus the value of alizarin red S as a screening test for BCP is questioned. In view of the variable sensitivity and specificity of different observers for MSUM and CPPD and the concentration threshold for reliable crystal identification, greater caution in the interpretation of synovial fluid analysis is advised, and recommendations for increased quality control are supported.

Synovial fluid analysis is an important investigation in the diagnosis of joint sepsis or a crystal related arthropathy. ${ }^{12}$ Polarised light microscopy was first used for the identification of monosodium urate monohydrate (MSUM) and calcium pyrophosphate dihydrate (CPPD) crystals in the early $1960 \mathrm{~s}^{34} \mathrm{It}$ rapidly became the preferred technique, recommended in standard textbooks, ${ }^{15}$ and is now in routine use in rheumatology units throughout the world. More recently basic calcium phosphates (BCP) such as hydroxyapatite have been identified in synovial fluids by a number of sophisticated techniques. ${ }^{6-8}$ These crystals are not usually visible in the light microscope but can be seen more easily if the synovial fluid is stained. ${ }^{9}$ The use of alizarin red $S$ has been recommended for the routine screening of synovial fluids for BCP. ${ }^{10}$

It has been shown that reporting the presence or absence of crystals in synovial fluids affects patient management. ${ }^{11}$ Despite this there have been very few published reports of any quality control or analysis of the validity of synovial fluid analysis.

\footnotetext{
Accepted for publication 16 January 1989

Correspondence to Professor Paul Dieppe. Rheumatology Unit. University Department of Medicine. Bristol Royal Infirmary. Marlborough Strect. Bristol BS2 8HW.
}

Two recent studies reported a considerable variation in the results from different laboratories examining the same fluids. ${ }^{12} 13$ The authors of these papers recommended the introduction of quality control measures, and also suggested that special technicians should be trained to carry out synovial fluid examinations.

This paper reports the results of an investigation into the sensitivity and reliability of six different observers. Varying concentrations of crystals were added to synovial fluids after aspiration so that the ability of different people to detect small or large quantities of crystals could be assessed.

\section{Materials and methods}

SYNOVIAL FLUIDS

Synovial fluid was obtained from the knee or shoulder joint of 13 patients requiring therapeutic aspirations. The clinical diagnoses of the patients were rheumatoid arthritis (eight patients, 10 synovial fluid samples) and osteoarthritis (five patients). No patient had received an intra-articular injection into the aspirated joint within the previous eight weeks. Fluids were transported to the laboratory in plain sterile containers, with no added anticoagulant or preservative, and examined within a few hours of 
aspiration in 12 cases and after refrigerated storage at $-20^{\circ} \mathrm{C}$ in four.

\section{PREPARATION OF CRYSTALS}

Synthetic crystals of MSUM were prepared by the method of Seegmiller et al, ${ }^{14}$ CPPD by the polyacrylamide gel method of Harries et al, ${ }^{15}$ and BCP by seeded aqueous growth. ${ }^{16}$ The crystals were examined by infrared spectroscopy for chemical purity and in the polarised light microscope. Samples with a similar morphology to those found naturally were used for the experiments. The MSUM crystals were needle shaped with a mean length of $5 \mu \mathrm{m}$; the CPPD was predominantly triclinic, brick shaped crystals, 2-20 $\mu \mathrm{m}$ long; and the BCP particles were carbonated apatite crystals, tending to clump into aggregates of about $0 \cdot 1-1 \mu \mathrm{m}$ diameter.

PREPARATION OF SLIDES

Crystal species (5 mg of each) were sonicated in saline to produce a well dispersed suspension, centrifuged, and the deposit resuspended in synovial fluid. The samples were mixed well and were further diluted with synovial fluid to produce preparations of differing crystal concentration. Two drops of each fluid were added to acetone washed microscope slides. Coverslips were put on and sealed with nail varnish. Each slide was renumbered, coded, and examined in a polarised light microscope. For BCP crystal identification $20 \mu \mathrm{l}$ of each synovial fluid preparation was mixed on a slide with $20 \mu \mathrm{l}$ of a filtered, $\mathrm{pH} 4 \cdot 2,2 \%$ alizarin red $\mathrm{S}$ stain before being sealed. Eleven slides were prepared from each synovial fluid; two with no added crystals, and one each with three different concentrations of the three types of crystal. Two additional unstained slides were prepared from synovial fluid preparations containing added $\mathrm{BCP}(100 \mu \mathrm{g} / \mathrm{ml})$. To ensure that the observers were unaware of the exact number of slides with each crystal concentration we randomly excluded nine unstained slides (eight with CPPD or MSUM, one preparation without added crystals) and five stained slides with apatite crystals.

\section{EXAMINATION OF SAM PLES}

Preliminary observations were made by the two people involved in sample preparation so that the most appropriate concentrations of added crystal and a scoring system for recording data could be developed. Each slide (total 166) was then examined by each of six observers who were unaware of the code, the number of samples with added crystals, or of each other's findings. Unstained samples were examined in a polarised light microscope with first order red compensator, magnification $\times 400$, for MSUM and CPPD crystals. Stained slides were examined at $\times 200$ in ordinary light for $\mathrm{BC} \overrightarrow{\vec{P}}$ identification. The unstained samples were score $\$$ separately for the presence or absence of MSUM and CPPD crystals and the stained slides wer⿳亠口冋. similarly recorded for BCP particles. When crystats were identified the result was graded as follows $1=$ occasional crystal(s) seen $(<1$ per microscop field); $2=$ at least one crystal per microscope field $3=\geqslant 10$ crystals per field for CPPD and MSUM and $\geqslant 5$ particles per field for BCP. The observers were two laboratory technicians (A and B), one witP specialist training and experience in crystal identification (A); a physician with little previous experience of synovial fluid analysis (C); two trainee rheume atologists (D and $E$ ); and a consultant rheumato logist with many years' experience of this technique (F).

At the beginning of the study all the observerg were shown examples of slides with each of the thres types of synthetic crystal. They were instructed in the identification of MSUM and CPPD crystals by morphology and birefringence using the polarise light microscope, and in the idenfication of $\mathrm{BC}$ particles stained with alizarin red $\mathrm{S}$ by ordinary ligh microscopy.

\section{Results}

CONCENTRATION OF CRYSTALS ADDED The preliminary investigations by two observer ( $A$ and $C$, and confirmed by $F$ ) indicated that considerable amount of crystalline material was necessary for the particles to be visible (Table 1). T8 obtain a score of 3 required $100 \mu \mathrm{g} / \mathrm{ml}$ of BCP of MSUM crystals and $1000 \mathrm{mg} / \mathrm{ml}$ of CPPD. Scores of 2 and 1 required respectively 10 - and 1000 -fol dilutions of MSUM and CPPD, and 100- and 1000 fold dilutions of BCP.

Table 1 Concentration of crystals in synovial fluid required to give three distinct scores when slides were examined by light microscopy

\begin{tabular}{|c|c|c|c|}
\hline \multirow[t]{2}{*}{ Crystal score* } & \multicolumn{3}{|c|}{ Crystal concentration $(\mu \mathrm{g} / \mathrm{ml})$} \\
\hline & $M S U M^{+}$ & $C P P D^{+}$ & $B C P^{\dagger}$ \\
\hline 3 & 100 & 1000 & 100 \\
\hline 2 & 10 & 100 & 1 \\
\hline 1 & $0 \cdot 1$ & 1 & $0.1 c$ \\
\hline
\end{tabular}

${ }^{*} 1=$ occasional crystals seen; $2=$ at least one crystal per microscope ficld: $3=10$ MSUM and CPPD crystals or $5 \mathrm{BCP}$ particles per field +MSUM=monosodium urate monohydrate: $C P P D=$ calciug pyrophosphate dihydratc: $\mathrm{BCP}=$ basic calcium phosphate. 
Table 2 Combined results of crystal examination by the six observers on synovial fluid samples without (16 slides) and with various concentrations of synthetic $M S U M^{*}$ (41 slides) and $C P P D^{*}$ (42 slides)

\begin{tabular}{lllll}
\hline $\begin{array}{l}\text { Crystal } \\
\text { added }\end{array}$ & $\begin{array}{l}\text { Concentration } \\
(\mu g / m l)\end{array}$ & $\begin{array}{l}\text { Total } \\
\text { number } \\
\text { of reports }\end{array}$ & $\begin{array}{l}\text { MSUM } \\
\text { positive } \\
(\text { No }(\%))\end{array}$ & $\begin{array}{l}\text { CPPD } \\
\text { positive } \\
(\text { No }(\%))\end{array}$ \\
\hline None & - & 93 & $9(10)$ & $44(47)$ \\
MSUM & $0 \cdot 1$ & 77 & $13(17)$ & $33(43)$ \\
& 10 & 84 & $73(87)$ & $17(20)$ \\
& 100 & 83 & $83(100)$ & $1(1)$ \\
CPPD & 1 & 70 & $1(1)$ & $39(56)$ \\
& 100 & 88 & $3(3)$ & $78(89)$ \\
& 1000 & 89 & $4(4)$ & $86(97)$ \\
\hline
\end{tabular}

${ }^{*} \mathrm{MSUM}=$ monosodium urate monohydrate; $\mathrm{CPPD}=$ calcium pyrophosphate dihydrate.

tAny slide not reported by an observer has been excluded.

SAMPLES WITH NO ADDED CRYSTALS Each observer examined 16 slides of synovial fluid without previously identified or added MSUM or CPPD crystals; a positive score was often recorded, especially for CPPD. There were considerable observer variations: positive reports ranging from a low of $3 / 16$ to a high of $12 / 16$. Almost all these 'false positives' were only graded 1 . The presence of added BCP on two of the 16 slides was not associated with more false positive results than the same synovial fluid samples without added BCP. It did not appear that any of these synovial fluid samples contained natural MSUM or CPPD crystals. The observers varied in the samples they reported as containing crystals, and MSUM or CPPD were not consistently reported to be present when other slides made from these synovial fluids were examined. In particular, slides with low concentrations of added crystals were often reported negative for CPPD and MSUM.

\section{SAMPLES WITH ADDED MSUM OR CPPD CRYSTALS}

Table 2 summarises the results recorded from the 83 slides to which varying concentrations of MSUM and CPPD crystals had been added. Correct identification improved with increasing crystal concentration in synovial fluid. Observer variation was apparent (Table 3). This table records the number of false negative and false positive scores for each of the six observers and shows their variable sensitivity and specificity for each crystal. There was much more variation between the observers in their sensitivity and specificity for CPPD than for MSUM. One observer, the experienced rheumatologist (F), stood out for low sensitivity and high specificity for CPPD. In general, specificity was much better for MSUM than for CPPD.

\section{ALIZARIN RED S STAINED SLIDES}

Sixty seven stained slides were examined by each observer. Most of these slides were scored positive for BCP, whether or not carbonated apatite crystals had been added to the synovial fluid. There was a wide range of recorded results and a lot of discrepancy between the different observers. Table 4 shows that only the slides with the highest amount of added BCP $(100 \mu \mathrm{g} / \mathrm{ml})$ were given higher scores than samples without added BCP by most of the observers, and some were given a low score.

Table 3 False negative (FN) and false positive $(F P)$ results recorded by the six observers examining 41 slides with MSUM* and 42 slides with CPPD, ${ }^{*}$ and the sensitivity and specificity of each observer for each crystal typet

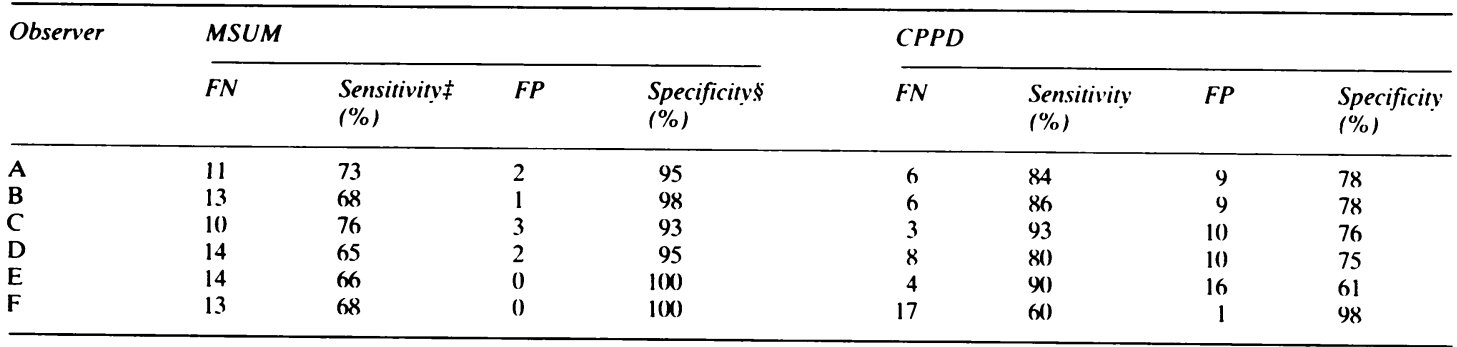

${ }^{*}$ MSUM = monosodium uratc monohydratc: $\mathrm{CPPD}=$ calcium pyrophosphatc dihydratc.

+Note: Any slide not reported by an observer has becn excluded from the analysis.

$\ddagger$ Sensitivity is the ratio of the observed positive (OP) to the expected positive results $=O P /(O P+F N)$.

$\$$ Specificity is the ratio of the observed negative $(O N)$ to the expected negative results $=O N /(O N+F P)$. 
Table 4 Results of the 67 alizarin red $S$ stained slides examined by the six observers for basic calcium phosphate $(B C \cdot \vec{P}$

\begin{tabular}{|c|c|c|c|c|c|c|}
\hline \multirow{2}{*}{$\begin{array}{l}\text { Concentration } \\
\text { of added } B C P \\
\text { (ug } m l)\end{array}$} & \multirow{2}{*}{$\begin{array}{l}\text { Number of } \\
\text { slide's }\end{array}$} & \multicolumn{5}{|c|}{ Number $(\%)$ of reports wilh $B C P$ score } \\
\hline & & () & $l$ & 2 & 3 & Vo resull \\
\hline 0 & 18 & $8(7)$ & $56(52)$ & $31(29)$ & $13(12)$ & $\ldots$ \\
\hline$(0 \cdot 1$ & 16 & $7(7)$ & $61(64)$ & $18(19)$ & $10(10)$ & - \\
\hline 1 & 16 & $5(5)$ & $48(50)$ & $32(33)$ & $10(10)$ & $1(1)$ \\
\hline $1(x)$ & 17 & $3(3)$ & $18(18)$ & $62(61)$ & $17(17)$ & $2(2)$ \\
\hline
\end{tabular}

\section{Discussion}

This study was designed to assess the reliability of synovial fluid analysis for crystals. We were particularly interested in the comparative results obtained from six observers with varying experience. and in ascertaining the threshold concentration of crystals which can be detected by standard techniques.

The initial experiments indicated that the threshold concentration for crystal identification was in the order of $0 \cdot 1-1 \mu \mathrm{g} / \mathrm{ml}$. The tests were therefore carried out using concentrations ranging from $0 \cdot 1$ to $1000 \mu \mathrm{g} / \mathrm{ml}$. The results show clearly that the chances of any of the observers making a correct" identification increased as the concentration of crystals in the fluids rose. Large numbers of false positives and false negatives were recorded at the lowest concentrations, indicating that reliable identification requires in the order of $10 \mu \mathrm{g} / \mathrm{ml}$ or more of crystals in the synovial fluid.

The amount of crystalline material in pathological specimens is difficult to calculate. It has been estimated that in chronic CPPD related arthritis the crystal concentration is in the order of $3 \mu \mathrm{g} / \mathrm{ml}$. increasing to $26 \mu \mathrm{g} / \mathrm{ml}$ in acute 'pseudogout'. and for BCP crystals, concentrations ranging from 3 to $18 \mu \mathrm{g} / \mathrm{ml}$ have been reported. ${ }^{\times}$Probably the number of free particles will vary considerably in different patients, and in many cases their quantity will be at. or even below, the threshold at which most observers are likely to be able to make a positive identification. This may be one explanation for the reported existence of acute gout in the absence of observed MSUM crystals, ${ }^{17}$ as well as the observation that crystals can sometimes be found by electron microscopy when they have not been observed by light microscopy. ${ }^{\text {Ix }}$

The data also show that there was less difficulty with MSUM than with CPPD crystals: the number of false negative and false positive results was lower. and there was less variation between the observers in the sensitivity and specificity for MSUM than for CPPD. One observer had a much higher specificity for CPPD than any of the others, though a lower sensitivity. This observer, the most experienced of

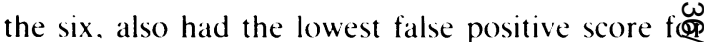
the synovial fluid without added CPPD and MSU and was the most likely to miss crystals at low concentrations. This suggests a different approaco to the examination and a greater reluctance to report occasional particles or equivocal finding Other differences were apparent, particularly in the recording of alizarin red S stained slides. Many of the observers reported that they found it difficult to score slides for BCP as the stained particles we unevenly distributed on the slides.

The reason for the large number of false positivo identifications, particularly for CPPD crystals. wi not obvious. Some of the fluids were stored be $\overrightarrow{R o}$ examination and could possibly have grown cry tide in vitro during that period. This has been repouget to occur, with the formation of brushite. whis could conceivably be confused with CPPD. Most of the fluids were examined fresh. howeve and star shapes indicative of in situ formation ${ }^{1 \times}$ we not seen. It therefore seems unlikely that in vition growth was responsible. Another possible explan tion might be that many of the fluids containes pathological CPPD. Against this are the facts the most fluids came from patients with rheumatok arthritis, who mat have a low incidence of chondræ calcinosis." different observers recorded false po: tives from different slides, and none of the observez. consistently reported CPPD crystals on other slid made from the same synovial fluid sample: slide with low concentrations of added crystals were ofteol reported negative for CPPD and MSUM. It seen more likely that many of these observations were contaminants or other crystalline material, such lipids and proteins. which looked like occasionof CPPD crystals. Such a finding has relevance to tho data of Fawthrop and colleagues. who reportad CPPD-like particles in a normal synovial fluid, ${ }^{21}$ and to reports of a high incidence of CPPD crystals osteoarthritis, even in the absence of chondre calcinosis. ${ }^{21}$ Two recent investigations compario the results from different laboratories both found that CPPD causes more problems than MSUM, as this study. 1213 These authors also reported fal 
positives, but much less frequently than in our investigation.

Our findings may not be directly relevant to the usual diagnostic situation for a number of reasons. Firstly, the observers knew that many of the fluids would have had crystals added. sometimes in small quantities. They may therefore have had an inappropriately low threshold for reporting positive findings. Clinicians often search fluids hard, however, to find one or two particles to 'confirm' their clinical diagnosis. A second difference was in the use of synthetic rather than natural crystals. These CPPD were larger and more irregular than the natural particles in many instances. Thus the synthetic crystals might have been confused with other contaminating birefringent material on the slides. CPPD crystals that form in synovial fluid in vivo, however, may vary considerably in size and shape. ${ }^{18} \mathrm{~A}$ small quantity of saline was added to the fluids with the crystals, potentially introducing other artefacts, but subsequent tests to see if saline addition induced the presence of birefringent material were negative.

The sensitivity for MSUM and CPPD in synovial fluid has been shown to be about $70 \%$ in both our study and a recent report.22 Despite previous publications by Schumacher" and Gatter ${ }^{2}$ stressing the need for good technique and the number of possible artefacts seen in the polarised light microscope, it is of considerable concern that trained observers often report false positives. some for urate as well as pyrophosphate crystals. ${ }^{12}{ }^{13}$ This and the previous studies suggest that polarised light microscopy cannot be regarded as a definitive investigation, and that great care must be taken in interpreting the results, particularly as they will often influence therapeutic decisions. ${ }^{11}$ Most false positive results in this study were graded 1 , indicating that only occasional particle(s) had been seen. Reports based on the detection of isolated birefringent particles should be treated with great caution.

It was not possible to test the intraobserver error as well as the interobserver differences. Even when slides are sealed with nail varnish they will slowly dry and change. Repeated observation by several observers would have extended the life of any individual slide from a period of only a few hours to one of several days, and it was thought likely that this would introduce more artefacts. It seems unlikely that the few hours between the first and last observations in the study influenced the results, and no order effect was apparent. Many of the slides were examined again by those who prepared them. and no significant drying or changes were noted in the first 48 hours. Further experiments to assess intraobserver error are therefore possible and would be valuable.
The results of the alizarin red S staining were not unexpected in view of our previous experience. ${ }^{20}$ In our laboratory the results from this investigation have never been as good as published results. Paul and colleagues found that concentrations as low as $0.005 \mu \mathrm{g} / \mathrm{ml}$ of apatite could be detected using this test. ${ }^{10}$ Some background staining is usual in synovial fluids, however, making detection of BCP more difficult. Bardin et al found apatite crystals by electron microscopy in most heavily staining samples, in agreement with previous investigations, but fluids with lesser degrees of alizarin red $\mathrm{S}$ staining particles rarely contained $\mathrm{BCP} .{ }^{23}$ The threshold for calling a sample positive needs to be high.

Although some authors do not consider that the presence of occasional crystals in synovial fluid is significant, ${ }^{24}$ others do. ${ }^{17}$ In view of this, and the knowledge that the presence of crystals changes clinical decision making. " the data have major implications for everyday rheumatological practice. They suggest that only a proportion of patients with crystal related arthropathies have a high enough concentration of particles in their fluids to allow confident identification. Many false negatives are probably being reported. It also indicated that false positives, particularly for CPPD crystals, may not be uncommon. The findings on stained slides seriously question the value of alizarin red $\mathrm{S}$ as an effective screening test for BCP crystals. Finally, the discrepancies between the sensitivity and specificity of different observers suggest that we need quality control and special training for this laboratory investigation. We recommend greater caution in the interpretation of synovial fluid analysis, and more attention to the sensitivity, specificity. and reliability of crystal identification in rheumatological practice.

We would like to thank the Arthritis and Rheumatism Council for financial support.

\section{References}

1 Schumacher H R. Synovial fluid analysis. In: Kelley W N. Harris E D. Ruddy S. Sledge C B. eds. Textbook of rheumatology. 3rd ed. Philadelphia: Saunders, 1987: 568-79.

2 Gatter R A. A practical handbook of joint fluid analysis. Philadelphia: Lea and Febiger, 1984.

3 McCarty D J. Hollander J L. Identification of urate crystals in gouty synovial fluid. Ann Intern Med 1961: 54: 452-60.

4 McCarty D J, Kohn N N. Faire J S. The significance of calcium pyrophosphate crystals in synovial fluid of arthritic patients: the pseudogout syndrome. I. Clinical aspects. Ann Intern Med 1962: 56: 711-37.

5 Vernon Roberts B. Synovial fluid and its examination. In: Scott J T. ed. Copeman's textbook of the rheumatic diseases. 6th ed. Edinburgh: Churchill Livingstone. 1986: 251-77.

6 Dieppe P A. Huskisson E C. Crocker P. Willoughby D A. 


\section{Gordon, Swan, Dieppe}

Apatite deposition disease: a new arthropathy. Lancet 1976; i: 266-9.

7 Schumacher H R, Smolyo A P, Tse R L, Maurer K. Arthritis associated with apatite crystals. Ann Intern Med 1977; 87: 411-6.

8 Halverson P B, McCarty D J. Identification of hydroxyapatite crystals in synovial fluid. Arthritis Rheum 1979;22:389-95.

9 Schumacher H R. Synovial fluid crystals: methods for identification and introduction to the clinical syndrome. In: Brooks P M, York J R, eds. Rheumatology 85. 1985: 195-9. (Excerpta Medica International Congress Series No 675.)

10 Paul H, Reginato A J, Schumacher H R. Alizarin red S as a screening test to detect calcium compounds in synovial fluid. Arthritis Rheum 1983; 26: 191-200.

11 Eisenberg J M, Schumacher H R, Davidson P K, Kaufman L. Usefulness of synovial fluid analysis in the evaluation of joint effusions: use of threshold analysis and likelihood ratios to assess a diagnostic test. Arch Intern Med 1984; 144: 715-9.

12 Schumacher H R, Sieck M S, Rothfuss S, et al. Reproducibility of synovial fluid analyses. Arthritis Rheum 1986; 29: 770-4.

13 Hasselbacher $P$. Variation in synovial fluid analysis by hospital fluid laboratories. Arthritis Rheum 1987; 30: 637-42.

14 Seegmiller J E, Howell R, Malawista S E. The inflammatory reaction to sodium urate. JAMA 1962; 180: 469-75.

15 Harries J E, Dieppe P A, Heap P, Gilgead J, Mather M, Shah $J$ S. In vitro growth of calcium pyrophosphate crystals in polyacrylamide gels. Ann Rheum Dis 1983; 42 (suppl): 100-1.
16 Nancollas G. The growth of crystals in solution. Advances Colloid and Interface Science 1979; 10: 215-52.

17 Schumacher H R, Jimenez S A, Gibson R, et al. Acute gou arthritis without urate crystals identified on initial examination of synovial fluid. Arthritis Rheum 1975; 18: 603-12.

18 Dieppe P A, Crocker P R, Corke C F, Doyle D V, Huskiss E C, Willoughby D A. Synovial fluid crystals. Q J Med 1979; 45 533-53.

19 Doherty M, Dieppe P, Watt I. Low incidence of calciu pyrophosphate dihydrate crystal deposition in rheumato arthritis, with modification of radiographic features in coexistent disease. Arthritis Rheum 1984; 27: 1002-9.

20 FawthropF, Hornby J, Swan A, Hutton C, Doherty M, Dieppe $\vec{B}$ A comparison of normal and pathological synovial fluid. $B r+$ Rheumatol 1985; 24: 61-9.

21 Gibilisco P A, Schumacher H R, Hollander J L, Soper K A Synovial fluid crystals in osteoarthritis. Arthritis Rheum 1985? 28: 511-5.

22 Schumacher H R, Sieck M, Clayburne G. Quality control ito synovial fluid analysis: development of a testing mechanisio [Abstract]. Arthritis Rheum 1988; 31: S101.

23 Bardin T, Bucki B, Lansaman J, Ortiz Bravo E, Ryckewaert Dryll A. Coloration par le rouge alizarine des liquides artictu laires. Rev Rhum Mal Osteoartic 1987; 54: 149-54.

24 Currey H L F, Vernon Roberts B. Examination of synovial fluid. Clin Rheum Dis 1976; 2: 149-77. 